\title{
Effects of Feeder Cells on the Primary Culture of Ovarian Cell Populations from Adult Japanese Medaka (Oryzias latipes)
}

\author{
Jun Hyung Ryu ${ }^{1, \dagger}$ and Seung Pyo Gong ${ }^{1,2, *}$ \\ ${ }^{1}$ Department of Fisheries Biology, Pukyong National University, Busan 48513, Korea \\ ${ }^{2}$ Department of Marine-Biomaterials and Aquaculture, Pukyong National University, Busan 48513, Korea
}

Received February 12, 2020

Revised February 12, 2020

Accepted February 28, 2020

\section{*Correspondence}

Seung Pyo Gong

E-mail: gongsp@pknu.ac.kr

\section{ORCID}

https://orcid.org/0000-0002-9358-9568

${ }^{\dagger}$ Current address: Institute of Marine and Environmental Technology, University of Maryland Baltimore County, Baltimore, MD, USA

\begin{abstract}
Fish ovarian germline stem cells (OGSCs) that have the abilities to selfrenew and differentiate into functional gametes can be used in various researches and applications. A main issue to be solved for effective utilization of fish OGSCs is the development of their stable in vitro culture condition, but only few researches about fish OGSC culture have been reported so far. In this study, in order to find the clues to develop the culture condition for OGSCs from Japanese medaka (Oryzias latipes), we tried to establish somatic cell lines as a candidate for the feeder cells and evaluated its supporting effects on the culture of ovarian cell populations from 0 . latipes. As the results, the somatic cell lines could be established only from the embryonic tissues among three tissues derived from embryos, fins and ovaries. Three embryonic cell lines were tested as a feeder cell for the culture of ovarian cell population and all three cell lines induced cell aggregation formation of the cultured ovarian cells whereas the feeder-free condition did not. Furthermore, a significant cellular proliferation was observed in the ovarian cells cultured on two of three cell lines. As a trial to increase the capacity of the cell lines as a feeder cell that supports the proliferation of the cultured ovarian cells, we subsequently established a stable line that expresses the foreign 0 . latipes fibroblast growth factor 2 (FGF2) from an embryonic cell line and evaluated its effectiveness as a feeder cell. The ovarian cells cultured on FGF2 expressing feeder cells still formed cell aggregates but did not show a significant increase in cellular proliferation compared to those cultured on non-transformed feeder cells. The results from this study will provide the fundamental information for in vitro culture of medaka OGSCs.
\end{abstract}

Keywords: feeder cells, in vitro culture, Japanese medaka, ovarian germline stem cells

\section{INTRODUCTION}

Ovarian germline stem cells (OGSCs) differentiate into oocytes before birth and thus, their existence in the postnatal ovaries is controversial in most mammalian species (Telfer et al., 2005). In contrast, the existence of OGSCs in adult fish ovaries has been demonstrated (Nakamura et al., 2010) and the production of offspring through transplantation of adult female-derived OGSCs has been achieved in zebrafish (Wong et al., 2011; Wong et al., 2013) and trout (Yoshizaki et al., 2010; Lee et al., 2016). Thus, fish OGSCs can be a useful resource for the study 
of developmental biology, genetics and biotechnology like primordial germ cells (PGCs) and spermatogonial stem cells (SSCs). However, their utilization in the fields has been restricted due to a lack of knowledge regarding their in vitro culture implying that the effort should be made to establish a stable fish OGSC culture system.

Previously, Wong et al. demonstrated that rainbow trout splenic feeder cells expressing zebrafish leukemia inhibitory factor (LIF) and zebrafish ovarian somatic feeder cells expressing zebrafish fibroblast growth factor 2 (FGF2), glial cell derived neurotrophic factor (GDNF), gonadal soma derived factor (GSDF) or LIF could not only maintain germline competency of zebrafish OGSCs but also support their growth in vitro (Wong et al., 2013). For Japanese medaka (Oryzias latipes), we, recently, used the method that coats solid surface with polymers to enhance the culture outcome of OGSCs. As a result, coating the dishes with polydopamine, a polymer generated from dopamine through oxidation, influenced positively on the adhesion, survival and potency maintenance of enriched OGSC populations under feeder-free culture condition (Jeong et al., 2019). However, this method was effective only for the maintenance of medaka OGSCs in culture, and it did not induce significant growth of them unlike the feeder-dependent culture system that was used for zebrafish OGSC culture. This suggests that it is necessary to evaluate the effects of feeder-dependent culture system on medaka OGSC culture to know the more optimal basis for establishing long-term culture of OGSCs in medaka model.

For this reason, this study was performed to investigate the effects of feeder cells on the primary culture of $O$. latipes ovarian cell population, which were enriched by percoll density gradient centrifugation and differential plating, to find the clues to develop the feeder-dependent culture system for medaka OGSCs. In order to do that, we first examined the optimal tissue source to be able to supply feeder cell lines stably and then the enriched ovarian cell populations were cultured on the established feeder cell lines to evaluate its effectiveness on cell proliferation. Additionally, a feeder cell line artificially expressing medaka FGF2 was prepared and its effectiveness to O. latipes ovarian cell culture was also evaluated.

\section{MATERIALS AND METHODS}

\section{Animals}

Adult Japanese medaka (O. latipes) was purchased from a local aquarium and reared in $20 \mathrm{~L}$ tanks at $26^{\circ} \mathrm{C}$. Fish were fed two or three times a day with a commercial diet for flounder larvae (EWHA, Busan, Korea) and the photoperiod was maintained at light for $14 \mathrm{~h}$ and darkness for $10 \mathrm{~h}$. For the experiments, adult females were separated from the others and starved for at least $24 \mathrm{~h}$. All experiments using fish were conducted in compliance of ethical guidelines from the Institutional Animal Care and Use Committee (IACUC) of Pukyong National University, which approved our research proposal (approval number: 2016-07).

\section{Primary culture of the cells derived from three tissues}

In order to establish feeder cell lines for in vitro culture of $O$. latipes ovarian cell populations, primary cultures were carried out with the cell populations from embryos, fins and ovaries. Embryos at stage 32 to 36 were disinfected with 70\% (v/v) ethanol (SK Chemicals, Sungnam, Korea) for $10 \mathrm{sec}$ and washed twice with Dulbecco's phosphate buffered saline (DPBS; Gibco, Grand Island, NY, USA). After the removal of chorion and egg yolk with syringe needles, an embryo was transferred into each well of $0.1 \%(\mathrm{w} / \mathrm{v})$ gelatin (Sigma-Aldrich, St. Louis, MO, USA)-coated 96-well culture plates (Thermo Scientific, Vernon Hills, IL, USA) filled with culture medium. Each embryo was dissociated by pipetting 15 times using a syringe equipped with 26 gauge needle and the dissociated cells were cultured. To obtain fins and ovaries, fish were anesthetized with $0.1 \%(\mathrm{v} / \mathrm{v})$ 2-phenoxyethanol (SigmaAldrich) and disinfected with $70 \%$ (v/v) ethanol. For fin cell culture, caudal fins were removed from fish and cut into approximately $2 \mathrm{~mm}^{2}$ using a surgical blade and tissue fragments were placed on $0.1 \%(\mathrm{w} / \mathrm{v})$ gelatin-coated $35 \mathrm{~mm}$ culture dishes (SPL Life Sciences, Pocheon, Korea). After pressing the tissue fragments with coverslips, they were cultured in culture media. To isolate ovarian cells, ovaries were washed twice with DPBS containing $1 \%(\mathrm{v} / \mathrm{v})$ penicillin and streptomycin (P/S; Gibco) and dissociated mechanically with sterile scissors. Subsequently, enzymatic dissociation was conducted in Leibovitz's L-15 medium (L15; Gibco) supplemented with $500 \mathrm{U} / \mathrm{mL}$ collagenase type I (Worthington Biochemical Corporation, 
Lakewood Township, NJ, USA) for $1 \mathrm{~h}$ at $28^{\circ} \mathrm{C}$. After dissociation, an equal volume of L15 containing $10 \%(\mathrm{v} / \mathrm{v})$ fetal bovine serum (FBS; Gibco) was added and the cell suspension was filtered on a $40 \mu \mathrm{m}$ cell strainer (Falcon, Corning, NY, USA) to remove debris. Finally, the cells were washed twice with DPBS containing $1 \%(\mathrm{v} / \mathrm{v}) \mathrm{P} / \mathrm{S}$ and then used for experiments. For culture, the ovarian cells isolated were resuspended in culture medium and seeded on $0.1 \%(\mathrm{w} / \mathrm{v})$ gelatin-coated culture dishes. Culture media were L15 containing 20\% (v/v) FBS and 1\% (v/ v) $\mathrm{P} / \mathrm{S}$ regardless of the type of cell populations. All cell populations were cultured at $28^{\circ} \mathrm{C}$ with an air atmosphere and culture medium was changed every two or three days. When the cells reached more than $80 \%$ confluency, they were subcultured at a ratio of 1:2 to 1:3.

\section{Preparation of $O$. latipes FGF2 expression vectors}

To obtain O. latipes FGF2 cDNA, total RNA was extracted from adult testes using RNeasy Plus Mini Kit (Qiagen, Valencia, CA, USA) and cDNA was synthesized from $1 \mu \mathrm{g}$ total RNA using GoScript reverse transcription system (Promega, Madison, WI, USA) according to the manufacturer's instructions. After PCR amplification of the CDS region, a target band ( $468 \mathrm{bp}$ ) was extracted using gel extraction kit (Genotech, Daejeon, Korea) and ligated into a vector of All in One ${ }^{\mathrm{TM}}$ PCR Cloning kit (Biofact, Daejeon, Korea) according to the manufacturer's instructions. Then, the insert-ligated vector was cloned into $E$. coli $\mathrm{DH} 5 \alpha$ and the clones were analyzed by a commercial sequencing service (CosmoGenetech, Seoul, Korea) after plasmid extraction using Plasmid Extraction Mini Kit (Favorgen, Pingtung, Taiwan). FGF2 cDNA from the clones that have same sequences with that in the NCBI GenBank database was subcloned into pcDNA5-Flag-Med25 (Addgene, Cambridge, MA, USA) using the restriction sites of Nhe I and Not I. The primer sequences and PCR conditions used in this study are listed in Table 1.

\section{Establishment of a stable cell line that expresses foreign FGF2}

An established embryonic cell line was transfected with $1 \mu \mathrm{g}$ FGF2 expression vectors using $1 \mu \mathrm{L}$ lipofectamine 2000 (Invitrogen, Carlsbad, CA, USA) and the transfected cells were selected by treating $600 \mu \mathrm{g} / \mathrm{mL}$ hygromycin (Invitrogen) for 4 weeks. The colonies formed were harvested using cloning cylinders (Corning Life Sciences, Corning, NY, USA) and cultured individually. After establishment of stable cell lines, FGF2 mRNA expression was confirmed by RT-PCR analysis. To detect the protein expression, a cell line, of which FGF2 mRNA expression was confirmed, was used. Cells harvested from a $100 \mathrm{~mm}$ culture dish (SPL Life Sciences) were washed twice with DPBS. Then cell lysates were obtained by a sonication in $1 \%(\mathrm{w} / \mathrm{v})$ triton X-100 (Sigma-Aldrich) in DPBS and protein concentration of the cell lysates was measured using Pierce BCA protein assay kit (Thermo Scientific) according to the manufacturer's instructions. The denatured protein was loaded on $15 \%$ acrylamide SDS gels, electrophoresed for $1.5 \mathrm{~h}$ at 100

Table 1. Primer sequences used in this study

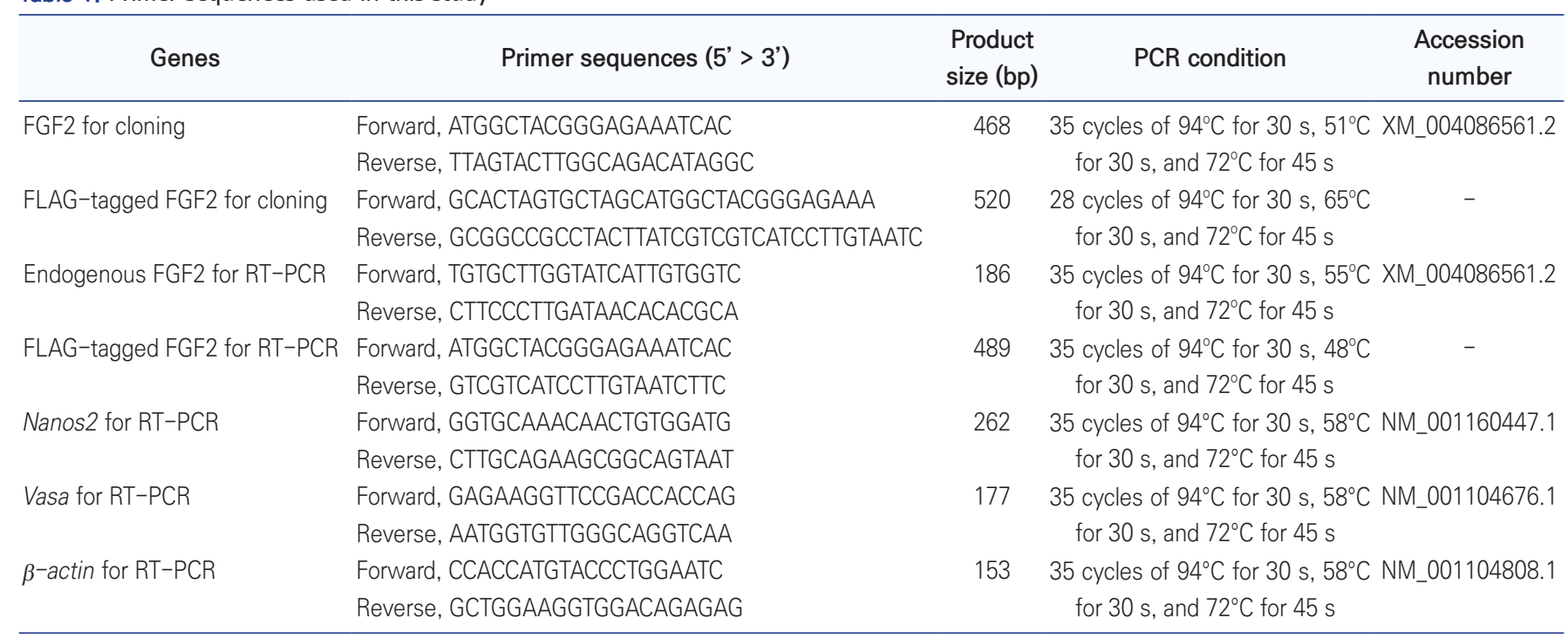


$\mathrm{V}$ and transferred to PVDF transfer membranes (Bio-Rad, Hercules, CA, USA) for $1 \mathrm{~h}$ at $100 \mathrm{~V}$. After blocking with $5 \%(\mathrm{w} / \mathrm{v})$ skim milk (Sigma-Aldrich) in PBS with $0.1 \%(\mathrm{v} / \mathrm{v})$ Tween 20 (Sigma-Aldrich) (PBST) for $1 \mathrm{~h}$ at room temperature, the membranes were incubated with monoclonal ANTI-FLAG $^{\circledR}$ M2 antibody (Sigma-Aldrich) at a 1:1,000 dilution for $15 \mathrm{~h}$ at $4^{\circ} \mathrm{C}$. After washing the membranes three times with PBST for 3 min, HRP-conjugated goat antimouse IgG secondary antibody (Thermo Scientific) was applied with 1:20,000 dilution for $1 \mathrm{~h}$ at room temperature. The membranes were washed three times with PBST for $3 \mathrm{~min}$ and signals of the proteins were visualized using enhanced chemiluminescence solution (Bio-Rad) and Xray films (Kodak, Tokyo, Japan) by exposure for $3 \mathrm{~min}$.

\section{Enrichment of germ cell populations}

To obtain ovarian cell populations, 10 ovaries were subjected to mechanical and enzymatic dissociation as mentioned in the part of ovarian cell isolation. Then, to enrich germ cell populations by removing red blood cells and anchorage-dependent cells, the retrieved cells were washed twice with DPBS containing $1 \%(\mathrm{v} / \mathrm{v}) \mathrm{P} / \mathrm{S}$ and loaded onto the top of layered Percoll (Sigma-Aldrich) solution consisting of $20,25,30,35,40,50$ and $60 \%$ in a $15 \mathrm{~mL}$ conical tube (Falcon). After centrifugation at $800 \times$ $g$ for 30 min, density fractions of $20-40 \%$ were harvested. After being washed twice with DPBS, the cells were resuspended in L15 supplemented with 10\% (v/v) FBS and $1 \%(\mathrm{v} / \mathrm{v}) \mathrm{P} / \mathrm{S}$. Then, $2-5 \times 10^{6}$ cells were seeded on a 35 $\mathrm{mm}$ petri dish (SPL Life Sciences) coated with $0.1 \%(\mathrm{w} /$ v) gelatin and incubated for $15 \mathrm{~h}$ at $28^{\circ} \mathrm{C}$. The cells that were floating or loosely bound were harvested and used for further experiments. These cells were designated as "enriched ovarian cell population" after this.

\section{In vitro culture of enriched ovarian cell populations}

To investigate the effect of feeder cells on the growth of ovarian cells, $1.5 \times 10^{4}$ live cells from the enriched ovarian cell populations were seeded on feeder cells or feeder-free condition. For the culture on feeder cells, 5 $\times 10^{5}$ feeder cells were seeded on a well of 24-well plates (SPL Life Sciences) and mitotically inactivated by treating $10 \mu \mathrm{g} / \mathrm{mL}$ mitomycin-C (Sigma-Aldrich) for $3 \mathrm{~h}$ before use. Prior to initiation of culture, all culture plates with or without feeder cells were incubated with ovarian germline stem cell medium (OGSM) for $24 \mathrm{~h}$ at $28^{\circ} \mathrm{C}$ in an air at- mosphere. Enriched ovarian cell populations were labeled with $6 \mu$ M PKH26 (Sigma-Aldrich) for $3 \mathrm{~min}$ and cultured in OGSM consisting of L15 supplemented with $25 \mathrm{mM}$ HEPES (Sigma-Aldrich), 6 mg/mL D-(+)-glucose (SigmaAldrich), 1\% (v/v) glutamax (Gibco), 1\% (v/v) non-essential amino acids (Gibco), 0.5\% (w/v) BSA (Sigma-Aldrich), 5\% (v/v) FBS, 0.25\% (v/v) trout serum (Caisson Laboratories, Smithfield, UT, USA), $1 \mu \mathrm{g} / \mathrm{mL}$ medaka embryo extract, $1 \%(\mathrm{v} / \mathrm{v}) \mathrm{P} / \mathrm{S}, 25 \mu \mathrm{g} / \mathrm{mL}$ bovine insulin (Sigma-Aldrich), $100 \mu \mathrm{g} / \mathrm{mL}$ recombinant human apo-transferrin (SigmaAldrich), $10 \mathrm{ng} / \mathrm{mL}$ recombinant human basic fibroblast growth factor (bFgf; Gibco), $10 \mathrm{ng} / \mathrm{mL}$ recombinant human glial cell-derived neurotrophic factor (Peprotech, Rocky Hill, NJ, USA), 5\% (v/v) Knockout serum replacement (Gibco), $50 \mu \mathrm{M}$ ascorbic acid (Sigma-Aldrich), 50 $\mu \mathrm{M} \beta$-mercaptoethanol (Gibco), and $2 \mathrm{nM}$ sodium selenite (Sigma-Aldrich) at $28^{\circ} \mathrm{C}$ in an air atmosphere. Medaka embryo extract was extracted as described previously (Choi and Gong, 2018). Half of the culture medium was replaced with fresh one every three days. For measurement of the cell number after culture for 10 days, the cells were harvested by treatment with $0.05 \%$ trypsin-EDTA (Gibco) and the number of fluorescent cells was counted using a hemocytometer (Paul Marienfeld GmbH \& Co. KG, Lauda-Königshofen, Germany) under a TS-100F microscope equipped with a fluorescent unit (Nikon, Tokyo, Japan).

\section{Statistical analysis}

The statistical analyses were performed using SPSS version 18 (IBM-SPSS, Chicago, IL, USA). The data were analyzed by One way-ANOVA or t-test followed by Duncan's method. Significant differences among groups were determined when $p<0.05$.

\section{RESULTS}

\section{Establishment of feeder cell lines}

To find optimal tissue source to be able to supply feeder cell lines, culture of the cells from three tissues including embryonic tissue, fin, and ovary was performed. All cell populations regardless of tissue sources showed primary adherence to substrates and of those, more than $80 \%$ were subcultured at least once (Table 2). However, cell death was observed after the removal of fin fragments and after 2 weeks of culture in fin- and ovary-derived cells, respec- 
Table 2. Culture outcomes of cell populations derived from three different tissues of Oryzias latipes

\begin{tabular}{|c|c|c|c|c|c|c|c|}
\hline \multirow{2}{*}{ Tissues } & \multirow{2}{*}{ Culture methods } & \multirow{2}{*}{ Trials } & \multirow{2}{*}{$\begin{array}{l}\text { No. }(\%)^{\dagger} \text { of cell populations } \\
\text { initially attached }\end{array}$} & \multicolumn{3}{|c|}{ No. $(\%)^{\dagger}$ of cell populations subcultured to } & \multirow{2}{*}{$\begin{array}{l}\text { No. }(\%)^{\dagger} \text { of cell populations } \\
\text { maintained } \geq 50 \text { days }\end{array}$} \\
\hline & & & & Passage 1 & Passage 5 & Passage 20 & \\
\hline Embryo & Dissociated cell culture & 27 & $27(100)$ & $22(82)$ & $18(67)$ & $9(33)$ & $22(81)$ \\
\hline Fin & Explant culture & 5 & $5(100)$ & $4(80)$ & $0(0)$ & $0(0)$ & $0(0)$ \\
\hline Ovary & Dissociated cell culture & 8 & $10(100)$ & $8(80)$ & $0(0)$ & $0(0)$ & $0(0)$ \\
\hline
\end{tabular}

${ }^{\dagger}$ Percentage of trials.

A

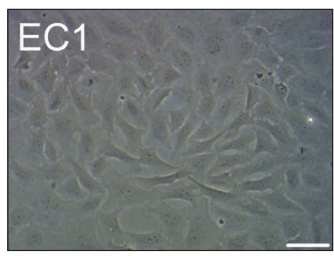

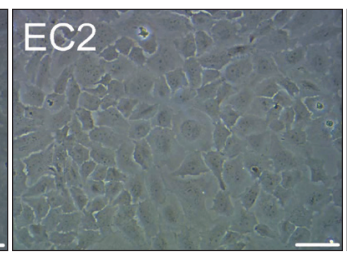

\section{EC3}

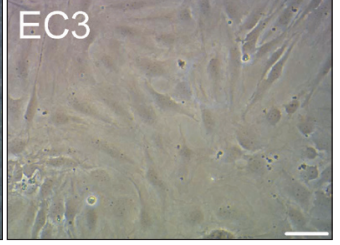

B

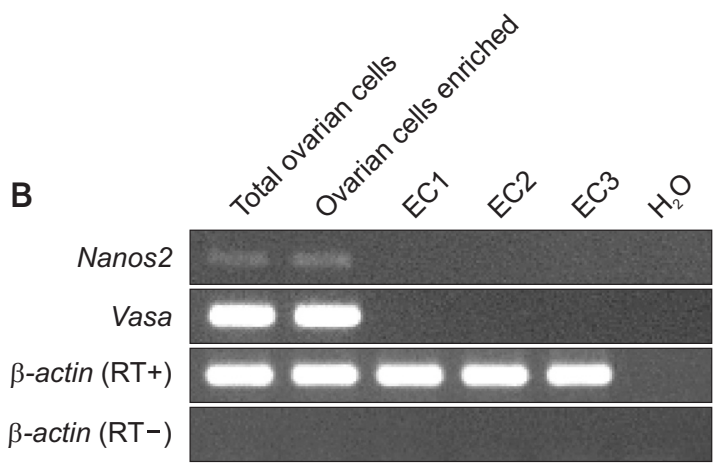

Fig. 1. Morphology and germline stem cell marker gene expression of the cell populations used in this study. (A) Cell morphology of embryonic cell lines (ECs) established. Each EC was derived from an embryo at stage 32 to 36. ECs were used as feeder cells for culture of enriched ovarian cell populations of Oryzias latipes after subculture over 30 times. In EC1 and EC3, fibroblast-like cells were predominant, while the majority of cells in EC2 were epithelial-like cells. Scale bar $=50 \mu \mathrm{m}$. (B) RT-PCR analysis of germline cell marker gene expression. The expression of germline stem cell-specific Nanos2 and germline cell-specific Vasa was detected in total ovarian cells and enriched ovarian cells but not in all ECs.

tively. The cell populations derived from fins and ovaries could not reach to passage 5 in culture and were just maintained for $18.2 \pm 6.0$ and $20.8 \pm 13.4$ days, respectively. On the contrary, 18 (66.7\%) out of 27 cell populations from embryonic tissues was cultured to more than passage 5 and $9(33.3 \%)$ out of 27 was cultured to more than passage 20 . Twenty two (81.5\%) out of 27 cell populations was maintained in culture for more than 50 days. These indicated that the embryonic tissues were an optimal tissue source to derive the cell lines that can be used as feeder cells among three tissues tested. Three embryonic cell lines (named as EC1, EC2, and EC3) were used as feeder cells in this study. These ECs contained two cell types that were morphologically different; fibroblast-like cells and epithelial-like cells. In EC1 and EC3, fibroblastlike cells were predominant whereas epithelial-like cells were predominant in EC2 (Fig. 1A). These three ECs did not express germline stem cell-specific Nanos2 and germ cell-specific Vasa genes indicating that germline cells were not included in these ECs (Fig. 1B).

\section{Effects of feeder cell lines on in vitro culture of enriched ovarian cell populations}

To examine the effects of feeder cells on the culture of ovarian cells, enriched ovarian cell populations that were labeled with fluorescent PKH26 were cultured on feeder cells derived from each of three ECs (EC1, EC2, and EC3) and the change of cell number was investigated after culture for 10 days. The enriched ovarian cell populations cultured on feeder cells formed cell aggregates unlike those cultured under feeder-free condition and those cultured on EC1 and EC3 showed a significant increase in cell number after culture for 10 days compared to those cultured on feeder-free condition ( $p<0.05$; Fig. 2).

\section{Effects of FGF2-expressing feeder cell line on in vitro culture of enriched ovarian cell populations}

The FGF2 expression vector was transfected into EC3 and stable cell line expressing FGF2 was subsequently established by antibiotic selection. RT-PCR analysis confirmed that the established cell line expressed the mRNA of foreign FGF2 whereas it did not express endogenous FGF2 (Fig. 3A). Moreover, the expression of FLAG-tagged 


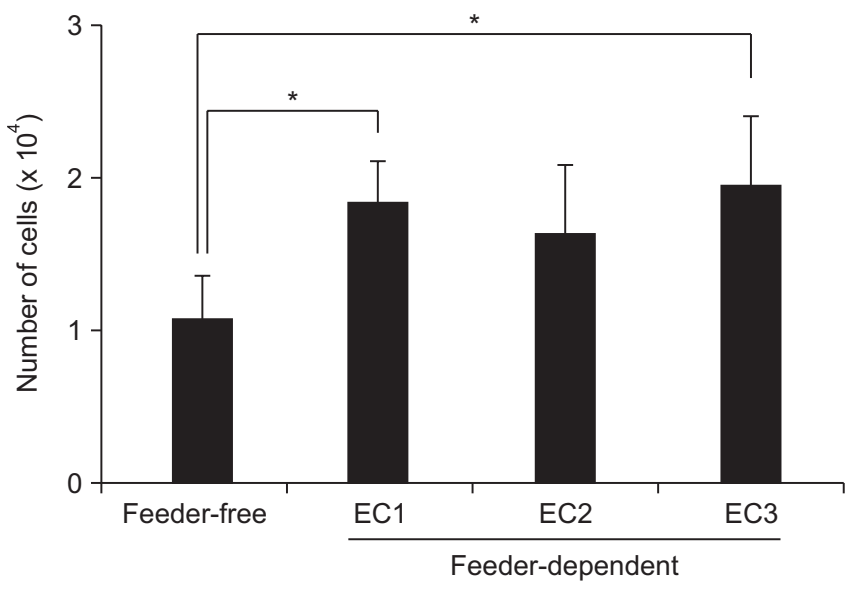

Fig. 2. Effects of feeder cells on the growth of enriched ovarian cell populations. Enriched ovarian cell populations were harvested by a combination of Percoll density gradient centrifugation and differential plating and labeled with $6 \mu \mathrm{M} \mathrm{PKH26.}$ Afterward, $1.5 \times 10^{4}$ enriched ovarian cells were co-cultured on three different feeder cell lines for 10 days at $28^{\circ} \mathrm{C}$ with an air atmosphere. After culture, the number of cells cultured on embryonic cell line (EC) 1 and EC3 were significantly higher than those cultured on feeder-free condition. The values were expressed as mean \pm SD. Asterisk $\left(^{*}\right)$ indicates significant differences, $p<0.05$.

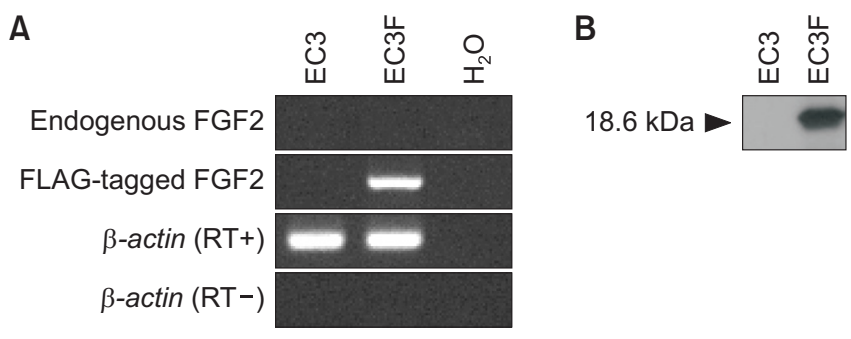

Fig. 3. Establishment of a feeder cell line that expresses foreign FGF2. The embryonic cell line 3 (EC3) derived from an embryo of Oryzias latipes was transfected with FLAG-tagged FGF2 expression vector and subjected to antibiotic selection. The colonies formed after antibiotic selection were individually picked and expanded to establish the stable cell line. (A) RT-PCR analysis of transgene mRNA expression in EC3-derived stable cell line expressing FGF2 (EC3F). Although endogenous FGF2 was not expressed in both EC3 and EC3F, the expression of FLAGtagged FGF2 mRNA was detected in the EC3F. EC3 cell line and $\beta$-actin were used as a negative control and an internal control, respectively. (B) Western blot analysis of transgene expression in EC3F. The expression of FLAG-tagged FGF2 (18.6 kDa) was detected in the EC3F. EC3 cell line was used as a negative control.
A

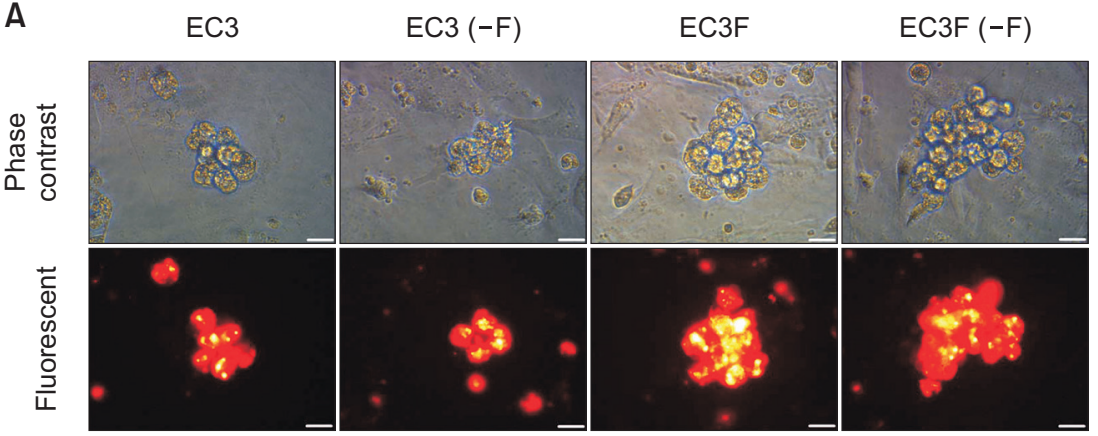

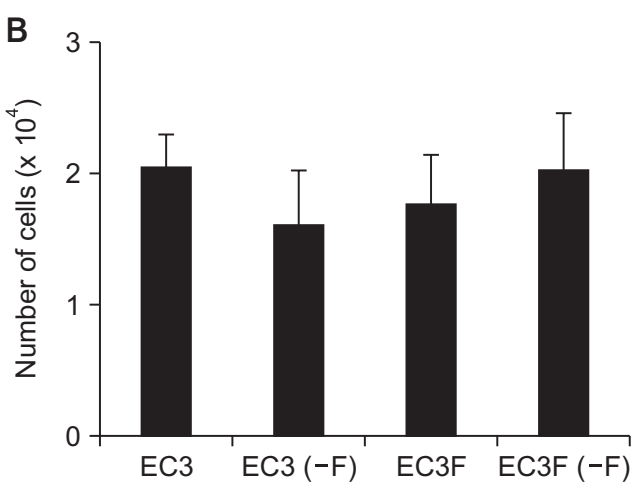

Fig. 4. Effects of EC3-derived stable cell line expressing FGF2 (EC3F) as the feeder cells on the growth of enriched ovarian cell populations. $1.5 \times 10^{4}$ cells of enriched ovarian populations that were labelled with PKH26 were co-cultured on embryonic cell line 3 (EC3) or EC3F as feeder cells for 10 days at $28^{\circ} \mathrm{C}$ with an air atmosphere. (A) Morphology of cell aggregates after culture. The formation of cell aggregates was observed in all treatment groups after culture. Scale bar $=20 \mu \mathrm{m}$. (B) Growth of enriched ovarian cell populations. All ovarian cells proliferated after culture regardless of treatment groups but no significant difference in cell number was detected among treatment groups. The values were expressed as mean \pm SD. $(-F)$ indicates that recombinant human bFGF was removed from the culture media.

FGF2 $(18.6 \mathrm{kDa})$ was detected in the stable cell line by western blot analysis (Fig. 3B). To test the effects of FGF2expressing feeder cell line (named as EC3F) on ovarian cell culture, enriched ovarian cell populations were cultured on EC3 or EC3F feeder cells. Furthermore, the effects of recombinant human bFGF as a medium supplement were also tested by removing it from OGSM. As the results, the cultured ovarian cells formed cell aggregates during in vitro culture regardless of treatment groups
(Fig. 4A). After culture for 10 days, the number of cells increased from 1.08 to 1.37 -fold relative to the initial cell number seeded according to the experimental groups (Fig. 4B). No significant difference was detected among experimental groups indicating that both human recombinant bFGF and medaka FGF2 produced by EC3F were not effective to induce ovarian cell proliferation. 


\section{DISCUSSION}

To develop a suitable culture condition for a specific cell type, several points including physical aspects, nutrients, signaling molecules such as hormones and cytokines, substrate condition and culture method should be considered (Freshney, 2010). Feeder cells have been often utilized to develop or improve the culture condition for certain cells, as they can supply several soluble signaling molecules and extracellular matrices to target cells during in vitro culture (Hongisto et al., 2012; Villa-Diaz et al., 2013). In mammals, the cells derived from embryos such as mouse embryonic fibroblasts have been frequently used for the culture of GSCs (Kanatsu-Shinohara et al., 2003; Kubota et al., 2004). In the present study, embryonic cell lines were established and two of them significantly promoted the growth of enriched ovarian cell populations when used as feeder cells. This would have been due to the extracellular matrices and cytokines secreted from the feeder cells. For establishment of embryonic cell lines, the embryos at stages 32 to 36 were used and they are known to express various kinds of hormones and growth factors such as GSDF and insulin (Assouline et al., 2002; Shibata et al., 2010). Based on this, the specific molecules that positively influenced to ovarian cells in culture needs to be found for the optimization of OGSC culture system.

To achieve more active proliferation of ovarian cells, a feeder cell line artificially-expressing FGF2 was developed. Because of the amino acid dissimilarities between mammals and fish, mammalian growth factors supplemented in culture media may have low or no activity on fish GSCs (Kawasaki et al., 2012). Thus, testing fish growth factors instead of mammalian ones is a major step for the establishment of a more suitable culture condition for fish OGSCs. FGF2, a member of the FGF family, is an important factor for the self-renewal of mammalian SSCs (Ishii et al., 2012; Takashima et al., 2015). The protein identity of FGF2 between human and medaka is $73 \%$ and it was reported that recombinant human FGF2 supports SSCs from fish including medaka, trout and zebrafish at 1-100 ng/mL concentrations (Hong et al., 2004; Shikina et al., 2008; Kawasaki et al., 2012). In addition, it was reported that zebrafish FGF2 supports the colony formation and proliferation of zebrafish SSCs and OGSCs during in vitro culture (Wong and Collodi, 2013; Wong et al., 2013). In our current study, culture of enriched ovarian cell populations on FGF2-expressing feeder cells did not show any significant promoting effects on cellular growth. This could be caused by the difference between species. Otherwise, the quantity of FGF2 secreted from the feeder cells might not sufficient to promote the growth of ovarian cells. Further experiment using the purified FGF2, the concentration of which can be adjusted, will be helpful. In addition, it may be necessary to use the feeder cells expressing multiple growth factors based on a previous report that showed that the feeder cells expressing two growth factors effectively promoted zebrafish OGSC proliferation compared to the feeder cells expressing a single growth factor (Wong et al., 2013).

In the present study, the effects of feeder cells on the culture of medaka ovarian cell populations were examined. The feeder cells derived from medaka embryonic tissues were able to support ovarian cell proliferation during short culture period. The results from this study will provide fundamental information for development of the optimal conditions for OGSC culture and will contribute to the utilization of OGSCs in various areas.

\section{CONFLICTS OF INTEREST}

No potential conflict of interest relevant to this article was reported.

\section{ACKNOWLEDGEMENTS}

This work was supported by a Research Grant of Pukyong National University (2019).

\section{AUTHOR CONTRIBUTIONS}

JH Ryu performed the experiments and wrote the paper; SP Gong conceived and designed the experiments and wrote the paper.

\section{AUTHOR'S POSITION AND ORCID NO.}

JH Ryu, Ph.D., Researcher, https://orcid.org/0000-0002-6738-5684

SP Gong, Professor, https://orcid.org/0000-0002-9358-9568 


\section{REFERENCES}

Assouline B, Nguyen V, Mahé S, Bourrat F, Scharfmann R. 2002. Development of the pancreas in medaka. Mech. Dev. 117:299-303.

Choi JH and Gong SP. 2018. Effects of temperatures and basal media on primary culture of the blastomeres derived from the embryos at blastula stage in marine medaka Oryzias dancena. J. Embryo Transf. 33:343-348.

Freshney RI. 2010. Culture of animal cells: a manual of basic technique and specialized applications. 6th ed, WileyBlackwell, Hoboken (NJ), pp. 11-23.

Hong Y, Liu T, Zhao H, Xu H, Wang W, Liu R, Chen T, Deng J, Gui J. 2004. Establishment of a normal medakafish spermatogonial cell line capable of sperm production in vitro. Proc. Natl. Acad. Sci. U. S. A. 101:8011-8016.

Hongisto H, Vuoristo S, Mikhailova A, Suuronen R, Virtanen I, Otonkoski T, Skottman H. 2012. Laminin-511 expression is associated with the functionality of feeder cells in human embryonic stem cell culture. Stem Cell Res. 8:97-108.

Ishii K, Kanatsu-Shinohara M, Toyokuni S, Shinohara T. 2012. FGF2 mediates mouse spermatogonial stem cell self-renewal via upregulation of Etv5 and Bcl6b through MAP2K1 activation. Development 139:1734-1743.

Jeong Y, Ryu JH, Nam YK, Gong SP, Kang SM. 2019. Enhanced adhesion of fish ovarian germline stem cells on solid surfaces by mussel-inspired polymer coating. Mar. Drugs 17:11.

Kanatsu-Shinohara M, Ogonuki N, Inoue K, Miki H, Ogura A, Toyokuni S, Shinohara T. 2003. Long-term proliferation in culture and germline transmission of mouse male germline stem cells. Biol. Reprod. 69:612-616.

Kawasaki T, Saito K, Sakai C, Shinya M, Sakai N. 2012. Production of zebrafish offspring from cultured spermatogonial stem cells. Genes Cells 17:316-325.

Kubota H, Avarbock MR, Brinster RL. 2004. Growth factors essential for self-renewal and expansion of mouse spermatogonial stem cells. Proc. Natl. Acad. Sci. U. S. A. 101:1648916494.

Lee S, Katayama N, Yoshizaki G. 2016. Generation of juvenile rainbow trout derived from cryopreserved whole ovaries by intraperitoneal transplantation of ovarian germ cells. Biochem. Biophys. Res. Commun. 478:1478-1483.

Nakamura S, Kobayashi K, Nishimura T, Higashijima S, Tanaka M. 2010. Identification of germline stem cells in the ovary of the teleost medaka. Science 328:1561-1563.

Shibata Y, Paul-Prasanth B, Suzuki A, Usami T, Nakamoto M, Matsuda M, Nagahama Y. 2010. Expression of gonadal soma derived factor (GSDF) is spatially and temporally correlated with early testicular differentiation in medaka. Gene Expr. Patterns 10:283-289.

Shikina S, Ihara S, Yoshizaki G. 2008. Culture conditions for maintaining the survival and mitotic activity of rainbow trout transplantable type A spermatogonia. Mol. Reprod. Dev. 75:529-537.

Takashima S, Kanatsu-Shinohara M, Tanaka T, Morimoto $\mathrm{H}$, Inoue $\mathrm{K}$, Ogonuki N, Jijiwa $\mathrm{M}$, Takahashi M, Ogura A, Shinohara T. 2015. Functional differences between GDNF-dependent and FGF2-dependent mouse spermatogonial stem cell self-renewal. Stem Cell Reports 4:489-502.

Telfer EE, Gosden RG, Byskov AG, Spears N, Albertini D, Andersen CY, Anderson R, Braw-Tal R, Clarke H, Gougeon A, McLaughlin E, McLaren A, McNatty K, Schatten G, Silber S, Tsafriri A. 2005. On regenerating the ovary and generating controversy. Cell 122:821-822.

Villa-Diaz LG, Ross AM, Lahann J, Krebsbach PH. 2013. Concise review: the evolution of human pluripotent stem cell culture: from feeder cells to synthetic coatings. Stem Cells 31:1-7.

Wong TT and Collodi P. 2013. Dorsomorphin promotes survival and germline competence of zebrafish spermatogonial stem cells in culture. PLoS One 8:e71332.

Wong TT, Saito T, Crodian J, Collodi P. 2011. Zebrafish germline chimeras produced by transplantation of ovarian germ cells into sterile host larvae. Biol. Reprod. 84:1190-1197.

Wong TT, Tesfamichael A, Collodi P. 2013. Production of zebrafish offspring from cultured female germline stem cells. PLoS One 8:e62660.

Yoshizaki G, Ichikawa M, Hayashi M, Iwasaki Y, Miwa M, Shikina S, Okutsu T. 2010. Sexual plasticity of ovarian germ cells in rainbow trout. Development 137:1227-1230. 